\title{
Zum Verhältnis von feiernder Gemeinde und Zuschauergemeinde am Bildschirm
}

\author{
von Wolfgang Fischer
}

In der jüngeren Diskussion über Sinn und Legitimität von Gottesdienstübertragungen ist immer stärker die unterschiedliche Teilnahmesituation der versammelten Gemeinde in der Kirche und des Zuschauers am Bildschirm in den Vordergrund getreten. Früher häufig vorgetragene Einwände gegen die mediale Vermittlung einer Meßfeier, vor allem der Verweis auf die Arkandisziplin oder die Profanierung des Heiligen, werden heute als untergeordnet eingestuft. Die Diskussion hat deutlich werden lassen, $\mathrm{da} ß$ die in der frühen Kirche geübte Praxis, Ungetaufte und Katechumenen von der Eucharistiefeier auszuschließen, im Widerspruch steht zu dem grundsätzlich öffentlichen Charakter des Gottesdientes. Ferner zeigen empirische Untersuchungen, daß Gottesdienstübertragungen fast ausschließlich von kirchlich orientierten Zuschauern verfolgt werden.

Nach wie vor umstritten ist unter Theologen, was Gottesdienstübertragungen überhaupt leisten können. Denn zum Wesen der Eucharistiefeier gehört die Versammlung der Gemeinde zu einer bestimmten Zeit und an einem bestimmten Ort. Die Zeitgleichheit zwischen dem sich ereignenden Gottesdienst in der Kirche und des vermittelten Gottesdienstes am Bildschirm vermag das Medium herzustellen, die räumliche Distanz kann es aber nicht überwinden, d.h. für den Rezipienten zu Hause ist die physische Teilnahme nicht möglich. Damit stellt sich die Frage, was überhaupt passiert, wenn Hunderttausende zur gleichen Zeit vor dem Bildschirm sitzen, um einen Gottesdienst mitzuverfolgen. Bleibt das nur die Summe von vielen religiösen Einzelakten oder bildet sich hier eine Art Gemeinschaft, vielleicht sogar eine Gemeinde? Sind die Christen am Bildschirm nur Beobachter eines liturgischen Geschehens oder sind sie in den Gottesdienst selbst einbezogen? Wie steht es um das Verhältnis von Zuschauer/in bzw. Zuschauergemeinde einerseits und feiernder Gemeinde in der Kirche andererseits? Die Beantwortung solcher oder ähnlicher Fragen ist Aufgabe der systematischen Theologie und der Liturgiewissenschaft, die Übertragungspraxis kann sie nicht lösen. Doch Erfahrungen aus der Praxis und empirische Erhebungen, reflektiert auf dem Hintergrund eines theologisch fundierten Gemeindeverständnisses, können Anstöße für die weitere Diskussion geben. Dazu sollen im folgenden einige Thesen formuliert werden.

\section{Die unterschiedliche Organisationsform von Ortsgemeinde und "Zuschauerge- meinde"}

Als wichtigstes Organisationsprinzip für die christliche Gemeinde hat sich im Verlauf der Kirchengeschichte das Territorialprinzip herausgebildet. Durch klar definierte Grenzen ist empirische unzweifelhaft faßbar, wer zu einer bestimmten Gemeinde gehört und wer nicht. Es gab in der Vergangenheit und Gegenwart immer wieder Versuche, christliche Gemeinden anders zu organisieren, z.B. die sog. Ordensgemeinden, 
die sich im 11. Jahrhundert um Reformprediger scharten, oder die sog. Basisgemeinden, die sich - angetrieben durch den gemeinsamen Einsatz für gerechtere soziale Verhältnisse - vor allem in Südamerika bildeten. Daß diese verschiedenen Gemeindeformen die Territorialgemeinde nie verdrängen konnten, liegt wesentlich an der guten Überschaubarkeit der Ortsgemeinde. Mit der sonntäglichen Eucharistiefeier in der für alle normalerweise leicht erreichbaren Kirche hat sie ein organisatorisches Zentrum. Daneben gibt es vielfache Möglichkeiten zur intensiven Kommunikation in Gruppen und durch die räumliche Nähe der Gemeindemitglieder auch die Möglichkeit zu nicht organisierten, direkten Interaktionen. Den Grad der Kommunikationsintensität bestimmt dabei der einzelne weitgehend selbst. Die Kommunikation wird dem Gemeindemitglied weder direkt noch indirekt aufgezwungen. Mit anderen Worten: Das Kommunikationsmodell "Territorialgemeinde « bestimmt sich durch die Faktoren räumliche Nähe und Möglichkeit zur Distanz. Dieses Spannungsverhältnis gewährleistet eine hohe Stabilität.

Eine völlig andere Organisationsstruktur hat die "Zuschauergemeinde«. Ihr Organisationsprinzip ist die Übertragung eines Gottesdienstes im Fernsehen. Sofern man hier überhaupt von Gemeinde im theologischen Sinn sprechen kann, worauf weiter unten noch näher eingegangen wird, handelt es sich um eine ad-hoc-Gemeinde. Sie organisiert sich nicht selbständig, sondern steht in Abhängigkeit von einer bestimmten Ortsgemeinde, die ihren Gottesdienst via Fernsehen für andere öffnet. Durch die groBe räumliche Distanz und mangelnde Überschaubarkeit ist eine direkte Kommunikation der Gemeindemitglieder untereinander nur sehr begrenzt möglich. Da jeder, der im Sendegebiet wohnt und ein entsprechendes Empfangsgerät besitzt, die Gottesdienstübertragung mitverfolgen kann, ist die Zuschauergemeinde der prinzipiellen Möglichkeit nach eine sehr offene Gemeinde.

In Wirklichkeit erweist sie sich aber als wesentlich homogener, als vielfach angenommen wird. Eine repräsentative Umfrage über die Nutzung religiöser Sendungen im Jahr 1981 zeigt deutlich, daß Gottesdienstübertragungen die Zuschauer selektieren. Wenn das Fernsehen eine Meßfeier ausstrahlt, so versammelt es nicht ein beliebiges Publikum am Bildschirm, vielmehr sprèchen diese Sendungen einen sehr bestimmten Personenkreis an. Für die Zuschauergruppe sind zwei Merkmale signifikant: hohes Alter und hohe kirchliche Identifikation.

$85 \%$ der Regelmäßig-Seher sind über 50 Jahre alt.

$50 \%$ dieser Gruppe geben an, daß sie noch regelmäßig zur Kirche gehen und

$27 \%$ der Befragten, die jede oder fast jede Gottesdienstübertragung mitverfolgen, gehen nach eigenen Angaben nie zur Kirche.

Das dürften vor allem die Hauskranken sein, die aus gesundheitlichen Gründen nicht mehr die sonntägliche Eucharistiefeier in der eigenen Gemeinde besuchen können. Die Daten lassen den Schluß zu, daß die Nutzung des Gottesdienstangebots im Fernsehen von der sonstigen Kirchgangspraxis abhängt. Die sog. »distanzierten « Christen werden kaum erreicht. Daraus läßt sich für unsere Fragestellung eine erste Bestimmung vornehmen:

- Das Verbältnis zwischen feiernder Gemeinde, deren Gottesdienst übertragn wird, und "Zuschauergemeinde" ist kein missionarisches, sondern ein solidarisches, den Glauben bestärkendes Verbältnis. 


\section{Der universalkirchliche Bezug der Ortsgemeinde}

Untersucht man das Verhältnis der Solidarität aus dem Blickwinkel der feiernden Gemeinde, die sich über das Medium für andere öffnet, so nimmt sie durch die Gottesdienstübertragungen etwas von ihrer universalkirchlichen Verantwortung wahr. Nach dem Verständnis des 2. Vatikanums ist Gemeinde ganz Kirche, aber eben doch nur für ihren Teil. Mit anderen Worten: Gemeinde steht in dem Spannungsverhältnis, einerseits sichtbares Zeichen und Vergegenwärtigung der Kirche zu sein, andererseits aber immer offen auf die Gesamtkirche hin zu sein. Ortsgemeinde ist ganz Kirche, sie ist aber nicht die Gesamtkirche. Fehlt diese dialektische Spannung, so ist für den einzelnen Kirche konkret nur noch schwer bzw. überhaupt nicht mehr erfahrbar oder die Gemeinde gleitet bei einer Überbetonung eines exklusiven Gemeinschaftsprinzips in ein Ghetto ab.

Bei der Gottesdienstübertragung wird exemplarisch die doppelte Bestimmung von Gemeinde deutlich: Auf der einen Seite feiert sie »ihren « Gottesdienst, Gemeinschaft mit Gott und untereinander wird für sie erfahrbar, auf der anderen Seite läßt sie via Bildschirm Mitchristen, die nicht zur eigenen Pfarrei gehören, an dieser Erfahrung teilhaben.

In der Praxis erweist sich die Öffnung auf Zuschauer hin nicht als Einbahnstraße. Durch diese Öffnung verändert sich etwas in der Gemeinde, sei es etwa die größere Sensibilität für Probleme alter und kranker Menschen oder sei es die Erfahrung einer Dankbarkeit vonseiten der Zuschauer. Die Gemeinde erfährt durch briefliche und telefonische Rückmeldungen der Zuschauer, daß beschädigtes Leben nicht weniger Leben, sondern mehr Leben sein kann. Kranke und Behinderte mit einem schweren persönlichen Schicksal schildern immer wieder, wie sie ihr Leben aus dem Glauben heraus meistern und welche Kraft sie aus dem Gebet schöpfen. In fast allen Briefen und Telefonaten kommt eine innere Zufriedenheit und eine Dankbarkeit für die Gottesdienstübertragungen zum Ausdruck.

Die Erfahrungen nach den Gottesdienstreihen im ZDF zeigen, daß das Fernsehen sehr viele Mitarbeiter in den Pfarreien mobilisiert. Die intensiven Vorbereitungen, die die mediale Vermittlung verlangt, wirkt sich fast immer auch langfristig für die Gemeinden aus. Nicht selten werden neue Mitarbeiter für die Gottesdienstvorbereitungsgruppen gefunden, Besuchsdienstgruppen oder Kommunionhelferkreise aufgebaut, die sonntags den Alten und Kranken die heilige Eucharistie bringen. Daraus ergibt sich die zweite These:

- Das Verhältnis von feiernder Gemeinde und Zuschauergemeinde ist ein komplementäres Verbältnis, bei dem sich beide gegenseitig befruchten. Sie verschmelzen nicht einfach zu einer Einheit, bzw. lassen sich nicht gleichsetzen, sind aber auch keine nebeneinander existierende, sich ausschließende Gebilde.

\section{Die Gottesdienstübertragung als Bezugspunkt}

Eine dritte Beschreibung ergibt sich, wenn man das Verhältnis von feiernder Gemeinde und "Zuschauergemeinde" unter dem verbindenden Aspekt betrachtet, also unter dem Aspekt des Gottesdienstes. In der Eucharistiefeier erfüllt die Gemeinde den Auftrag des Auferstandenen, seinen Tod und seine Auferstehung zu feiern. Die Menschen versammeln sich, weil sie sich von Gott gerufen wissen. Dieser Ruf ergeht aber an alle, 
also auch an Kranke und Behinderte. Wenn sie den Gottesdienst am Bildschirm mitvollziehen, so ist das eine der ihnen möglichen Antworten auf die Einladung des Auferstandenen. Deshalb handelt es sich bei dem Mitvollzug am Fernsehgerät nicht um einen rein individuellen religiösen Akt, sondern um eine wirkliche Teilnahme, die freilich qualitativ anders ist als die Teilnahme in der Kirche. Es entsteht eine Art Gemeinschaft sowohl zwischen den Menschen am Bildschirm und den Menschen in der Kirche als auch zwischen den "Zuschauern « untereinander. Die Brücke dazu schafft Christus selbst durch seinen Ruf. Der geistige Mitvollzug der Messe am Bildschirm macht den Kranken zwar nicht zu einem Mitglied der lokal vrsammelten Gemeinde, aber er bezieht sich auf den »jetzt« stattfindenden Gottesdienst.

\section{Daraus ergibt sich These 3:}

- Das Verbältnis zwischen Zuschauergemeinde und Ortsgemeinde ist ein Verbältnis der Relation, und nicht der Identifikation.

\section{Ist die Zuschauergemeinde eine wirkliche Gemeinde?}

Abschließend bleibt noch die Frage zu klären, ob bei der Zuschauergemeinde im streng theologischen Sinn von einer echten Gemeinde gesprochen werden kann. Aus dem bisher Gesagten ist deutlich geworden, daß die Zuschauergemeinde sich immer nur in Abhängigkeit von einer sich versammelnden Gemeinde bilden kann. Außerdem sind die direkten Kommunikationsmöglichkeiten der Mitglieder dieser »Gemeinde « untereinander praktisch nicht gegeben oder höchstens sehr begrenzt vorhanden. Da die direkte Kommunikationsmöglichkeit aber ein Wesensmerkmal für die christliche Gemeinde ist - dies gilt sowohl für das gottesdienstliche Handeln sowie für andere kirchliche Dienste - kann bei den Zuschauern nicht von einer Gemeinde im eigentlichen Sinn gesprochen werden. Andererseits ist aber die Zuschauerschaft bei einem Gottesdienst mehr als nur die Summe vieler einzelner Christen, die sich getrennt voneinander vor dem Bildschirm einfinden. Denn Christus schafft eine Verbindung, so daß eine geistig-geistliche Gemeinschaft entsteht. Als Getaufter gehört er wie die Mitglieder der feiernden Gottesdienstgemeinde vor Ort zum Volk Gottes.

\section{Praktische Konsequenzen}

Die Überlegungen zum Verhältnis zwischen Zuschauer- und Gottesdienstgemeinde erfordern einige praktische Konsequenzen für die Konzeption der Gottesdienstübertragungen:

- Die Anrede der Menschen am Bildschirm darf nicht eine Gleichsetzung mit der lokal versammelten Gemeinde assoziieren, darf aber andererseits auch nicht den Eindruck erwecken, als handle es sich bei den Versammelten vor dem Fernsehgerät nur um außenstehende Beobachter eines liturgischen Geschehens. Man wird Termini wählen müssen, die sich zwischen diesen beiden Polen bewegen.

- Das komplementäre Verhältnis von Gemeinde, aus der übertragen wird, und am Bildschirm versammelten Christen legt die Schaffung von Rückkanälen nahe. Bewährt hat sich etwa in diesem Punkt die ZDF-Konzeption, die nach jeder Übertragung einen Telefon- und Briefdienst vorsieht, so daß die Kranken zuhause mit Mitgliedern der »Fernsehgemeinde Kontakt aufnehmen können. 
- Da die Zuschauergemeinde kein interaktionsfähiges Handlungssystem und damit keine Gemeinde im vollen Sinne darstellt, ist eine Einbindung der Gottesdienstübertragungen in die lokale Krankenseelsorge der Pfarreien notwendig. Das Fernsehen kann die Kranken- und Altenpastoral nicht ersetzen. Es kann sie stützen, intensivieren und ergänzen, aber Priorität muß die Seelsorge der eigenen Ortsgemeinde des Zuschauers haben. Um das deutlich zu machen und nicht den Eindruck zu erwecken, als sei die Ortsgemeinde nur für die Gesunden und das Fernsehen für die Kranken zuständig, ist der Aufbau von Besuchsdiensten und Kommunionhelfergruppen sinnvoll. Wenn Eucharistiehelfer an Sonn- und Feiertagen, und damit auch an den Übertragungstagen, aus dem Gemeindegottesdienst ausgesandt werden, wird diese Priorität der Ortspfarrei deutlich.

\section{Summary}

A locally assembled community for the liturgical service is different from a TV audience watching this service. The relation between both is according to the experience with the German ZDF network that of solidarity and strengthen the faith rather than one of a missionary sending approach. Both groups of participants do not exist independet from each other. Their relation, however, is not one of identification. From this consideration there are practical consequences for the concept for the transmission of TV services.

\section{Résumé}

Une communauté réunie dans une salle pendant une messe se differencie d'une communauté de spectateurs, qui n'est pas dans une salle. D'après l'expérience de la ZDF, le rapport de deux, l'un de l'autre est plus un rapport de solidarité et de renforcement de croyance qu'une conscience missionaire. Les deux groupes de participants n'existent pas indépendament l'une de l'autre mais plûtot se complètent l'un l'autre; c'est le rapport de liaison et non celui d'une identification. A partir de ces raisonnements, il s'ensuit des déductions pour la pratique dans la conception de transmission de messe.

\section{Resumen}

Una comunidad de los fieles durante la misa se distingue de telespectadores de una misa. La relacion entre las dos comunidades consiste segun las experiencias del ZDF mas en solidaridad y firmeza de la fe que en una mision. Los dos grupos de participantes no existen por separado, pero se completan; es una relacion y no es una identificacion. De estas consideraciones resultan conclusiones para la practica de conceptuar transmisiones de misas. 